\title{
Spatially Coherent Activation Maps for Electrocardiographic Imaging
}

\author{
Josselin Duchateau*, Mark Potse, and Remi Dubois, Member, IEEE
}

\begin{abstract}
Objective: Cardiac mapping is an important diagnostic step in cardiac electrophysiology. One of its purposes is to generate a map of the depolarization sequence. This map is constructed in clinical routine either by directly analyzing cardiac electrograms (EGM) recorded invasively or an estimate of these EGMs obtained by a non-invasive technique. Activation maps based on noninvasively estimated EGMs often show artefactual jumps in activation times. To overcome this problem we present a new method to construct the activation maps from reconstructed unipolar EGMs. Methods: On top of the standard estimation of local activation time from unipolar intrinsic deflections, we propose to mutually compare the EGMs in order to estimate the delays in activation for neighboring recording locations. We then describe a workflow to construct a spatially coherent activation map from local activation times and delay estimates in order to create more accurate maps. The method is optimized using simulated data and evaluated on clinical data from 12 different activation sequences. Results: We found that the standard methodology created lines of artificially strong activation time gradient. The proposed workflow enhanced these maps significantly. Conclusion: Estimating delays between neighbors is an interesting option for activation map computation in ECGi.
\end{abstract}

Index Terms-Electrocardiography, Electrophysiology, Inverse problems,

\section{INTRODUCTION}

$\mathrm{C}$ ARDIAC arrhythmia occurs when the depolarization front propagates abnormally in the heart muscle. As electrical activity commands muscular contraction, electrical disorders can lead to a variety of symptoms, ranging from palpitations to sudden cardiac death.

Activation mapping is an important diagnostic procedure to understand such arrhythmias. In routine clinical work construction of the maps requires moving a catheter in contact with the endocardial or epicardial surface, and measuring electrogram (EGM) latencies in a point-by-point fashion. EGM latencies, corresponding to local activation time (AT),

\footnotetext{
"Manuscript received October 17, 2015. This work was supported by the French Agence Nationale de la Recherche (Grant IHU LIRYC ANR-10IAHU-04).

${ }^{*} \mathrm{~J}$. Duchateau is a student at Bordeaux University, France and resident at CHU de Bordeaux, France. He is with the IHU-LIRYC, Pessac, France (correspondence e-mail: josselin.duchateau@ihu-liryc.fr).

M. Potse is with the IHU-LIRYC, Pessac, France; Inria Bordeaux SudOuest, Talence, France; and the Center for Computational Medicine in Cardiology, Institute of Computational Science, Università della Svizzera italiana, Lugano, Switzerland.

R. Dubois is head of the signal processing team at the IHU-LIRYC, Pessac, France and an associate professor at ESPCI-ParisTech, Paris, France
}

are approximated either from a unipolar signal, at the point of maximum negative slope (intrinsic deflection time - IDT), or from a local bipolar signal, at the point of maximal signal amplitude [1].

This point-by-point technique has several important drawbacks: it is time-consuming, requires the mapped activation pattern to be repetitive and hemodynamically tolerated in order to allow mapping of the entire cavity, and is invasive.

Electrocardiographic imaging (ECGi) addresses some of these issues. Using a high density electrode vest to record the electrical potential on the body surface and image-based heart and torso geometries, the electrical potential on the epicardial surface is estimated [2]. This theoretically allows the reconstruction of the electrical activity of the entire heart for a single beat, in a non-invasive fashion. This computation, however, requires solving an ill-posed inverse problem and regularization of the solution.

The electrical conductivity of the torso results in a strong low-pass filtering effect on the measured potentials [3]. The regularization process also has the effect of removing high frequency components [4], and the overall computational workflow results in the reconstruction of strongly smoothed epicardial potentials, common to all potential-base inverses.

In this context, the determination of AT using a similar methodology as that of the contact EGMs is imprecise, as the intrinsic deflection is a high frequency feature of the signal.

To avoid this issue, research groups have focused on different approaches to solve the inverse problem of the ECG directly in terms of activation times. These methods are promising, but require additional assumptions [5]-[9]. In such formulations, segmentation of the ventricular endocardium is also needed. This requires either an MRI or the injection of a contrast agent during $\mathrm{CT}$, and is therefore a drawback to clinical implementation. Furthermore, the potential formulation of the inverse problem is more versatile because it also provides information concerning voltage amplitudes and EGM morphologies [10].

This paper is constructed in two parts. First a systematic evaluation of the precision of the standard measure (IDT) in the context of reconstructed EGMs from ECGi is undertaken. Then a method is proposed to enhance ECGi AT maps using estimated time delays between neighboring points.

The methodology is developed and parameters are adjusted using data from an in-silico model. The final validation is carried out on clinical data. 


\section{DATASETS}

\section{A. Model data}

Simulations were conducted using a monodomain reactiondiffusion heart model coupled to a bidomain torso model. Transmembrane currents were computed with the TNNP ventricular myocyte model [11]. The heart model consisted of left and right ventricles, with a $0.2 \mathrm{~mm}$ spatial resolution, and an anisotropic conduction derived from rule-based fiber orientation. The simulated transmembrane currents were conducted through the torso model at $1 \mathrm{~mm}$ resolution [12]. The torso model used had heterogeneous conductivity, with anisotropic skeletal muscle, lungs, and intracavitary blood. Cardiac and thoracic anatomy was based on MRI data.

Six different activation patterns were simulated on the same model, representing different pacing locations in the ventricles. Simulations were performed with the propag-5 software [12], [13] and ran on an IBM BlueGene/Q computer at the Institute for Development and Resources in Intensive Scientific Computing (IDRIS), France.

Simulated transmembrane potential upstrokes of adjacent epicardial cells were used as gold standard activation times on the epicardial surface. Gold standard delays between neighboring points were computed simply by using the difference in gold standard activation times.

252 body surface electrode recordings were simulated and the inverse problem was solved on a simplified open epicardial mesh comprising 1607 vertices and 3130 faces.

\section{B. Clinical data}

Clinical data comprising 12 activation sequences from 10 different patients were collected from the clinical electrophysiology laboratory of Hôpital du Haut Lévèque, Bordeaux, France. The dataset was constructed from patients admitted for epicardial ablation of ventricular tachycardia or ventricular fibrillation. Recorded ventricular rhythms were paced $(n=3)$ or conducted sinus beats $(n=9)$.

Torso and heart geometries were acquired using computed tomography (CT), and ECG recordings were then obtained using a 252-electrode vest (ECVUE, CardioInsight Inc., Cleveland, $\mathrm{OH}$ ) immediately prior to an invasive percutaneous epicardial mapping procedure. Epicardial and torso meshes were segmented using semi-automatic commercial software (CardioInsight Inc., Cleveland, OH).

The invasive data were recorded along with epicardial geometry using a clinical electro-anatomical mapping system (CARTO, Biosense Webster Inc., Diamond Bar, CA).

Gold standard activation times were provided by the CARTO system algorithm, which uses bipolar EGM amplitudes to compute ATs. Maps were corrected manually if necessary by an expert electrophysiologist.

The sparse CARTO activation time measures were interpolated on the CT epicardial mesh to create a dense map using a Shepard inverse distance weighing method [14] following a geodesic distance metric. Points on the mesh more than $2.5 \mathrm{~cm}$ away from the nearest activation time measurement were discarded from analysis.

\section{Methods}

\section{A. $E C G i$}

ECGi methodology has been described previously [2]. Briefly, this methodology uses epicardial potentials as an equivalent source to the heart's electrical activity. The torso is estimated to be a homogeneous conductor. The mathematical link between epicardial potentials and torso measurements (transfer matrix) is constructed using the method of fundamental solution (MFS). Regularization of the problem is done using standard Tikhonov on the MFS coefficients. The regularization parameter was chosen using the CRESO method.

The same methodology of inverse problem resolution was applied to the simulation data and the clinical data.

\section{B. Intrinsic deflection}

In contact mapping, the AT is determined on a unipolar signal at the time point corresponding to the IDT (Figure 1). If $s_{i}(t)$ is the signal at point $X_{i}$ at time $t$, the IDT $\widetilde{T}_{i}$ is defined by [1]:

$$
\widetilde{T}_{i}=\underset{t \in[0, T]}{\arg \min } \frac{d s_{i}(t)}{d t}
$$

In the ECGi workflow, a triangulated closed surface mesh of the epicardium is constructed from CT data. Solving the inverse problem results in the reconstruction of unipolar EGMs at each of the nodes of this mesh. The standard method of activation map calculation is to apply equation (1) directly to the reconstructed EGMs, directly yielding a dense surface activation map.

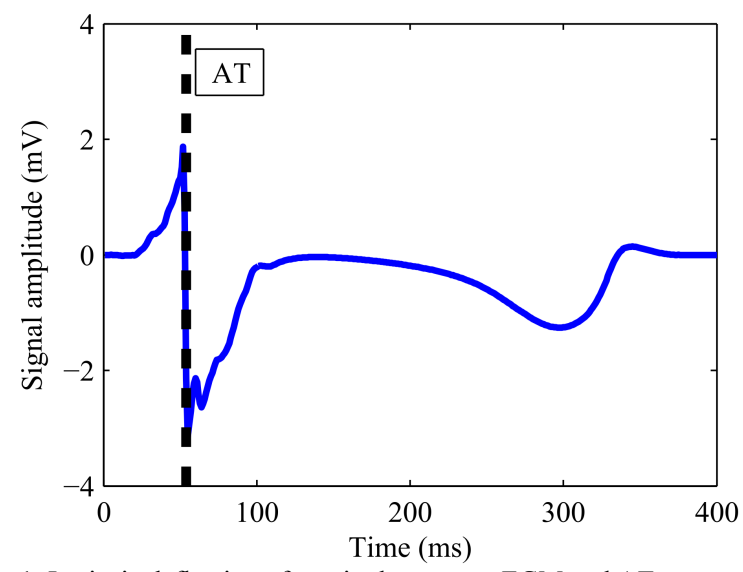

Figure 1: Intrinsic deflection of a unipolar contact EGM and AT.

\section{Confidence score}

IDT is therefore a good marker of activation time when signals contain a single sharp deflection. When signals are fragmented (several deflections), it is unclear which deflection corresponds to AT. Signals with weak IDTs are also imprecise, as ambiguity exists as to which time point of the IDT corresponds to AT [15]. 
We express these observations by adding to each marker a confidence score $\kappa_{i}$ defined as

$$
\kappa_{i}=\left|\frac{d s_{i}\left(\tilde{T}_{i}\right) / d t}{\int_{D} d s_{i}(t)}\right|
$$

where

$$
D=\left\{t \in[0, T], \frac{d s_{i}(t)}{d t}<0\right\}
$$

and $T$ is the QRS duration $(104 \pm 28 \mathrm{~ms}$ on our clinical dataset). Hence, in signals where the intrinsic deflection slope is large compared to the sum of the descending portions, this score will be high. It rapidly decreases when the intrinsic deflection is weak or the signal is fragmented (Figure 2).

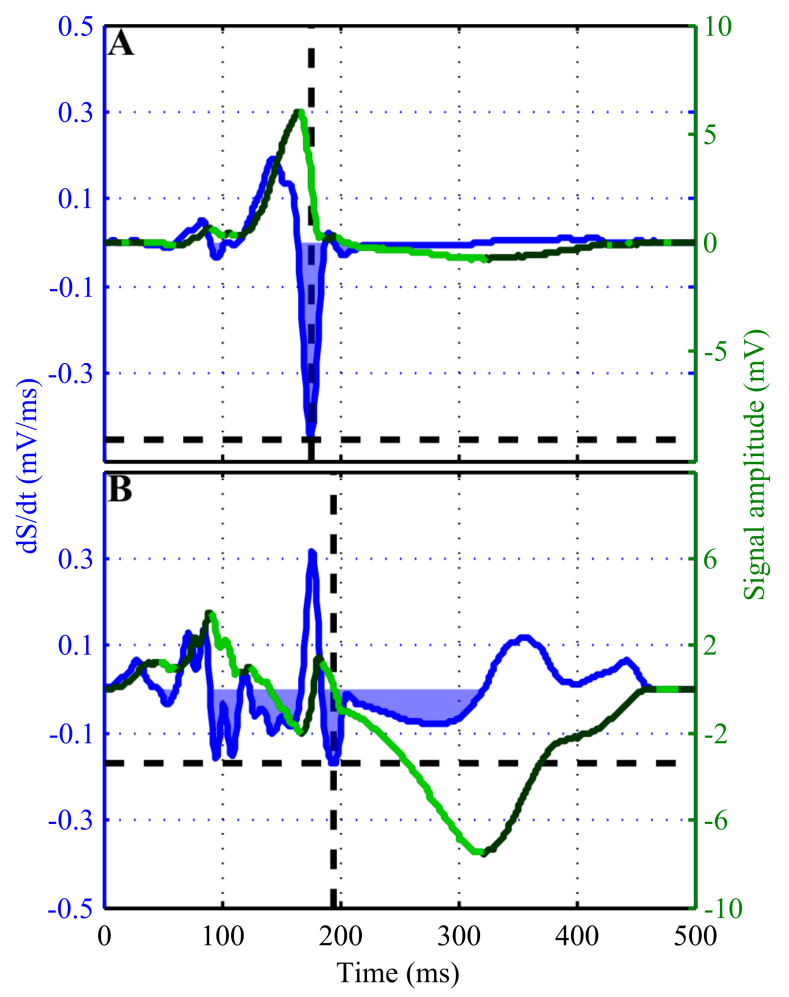

Figure 2: Example non-ambiguous (top) and ambiguous (bottom) signals with respect to AT estimation by IDT. Confidence score $\kappa_{i}$ is defined by dividing the slope of the maximal downstroke (dashed line) by the summed slope of descending portions (blue area)

\section{Signal delay computation}

In this paper, we propose to enhance activation map accuracy using estimated signal delays between neighboring (first order neighbors) epicardial nodes. Computation of such delays may indeed remain valid in the presence of fragmentation.
These delays can be computed in 3 different ways. An immediate approach is to compare estimated ATs directly.

$$
\tilde{\tau}_{i j}=\widetilde{T}_{j}-\tilde{T}_{i}
$$

An other method has been proposed using cross correlation [16], modeling the signal as a time-shift of its neighbor.

$$
\tilde{\tau}_{i j}=\underset{\delta \in[0 ; T]}{\arg \max } \int_{t=0}^{T}\left[s_{i}(t+\delta) s_{j}(t) d t\right]
$$

We propose to use a method described by Shors et al. [17] based on cross correlation of the signals' time differentials.

$$
\tilde{\tau}_{i j}=\underset{\delta \in[0 ; T]}{\arg \max } \int_{t=0}^{T}\left[\frac{d s_{i}(t+\delta)}{d t} \frac{d s_{j}(t)}{d t} d t\right]
$$

Numerical computation of the above delay yields a result with a temporal resolution equivalent to that of the signal's sampling rate $(1 \mathrm{kHz})$. As delays between neighboring nodes are small, a higher temporal resolution solution is desirable.

To obtain such a result, we used an approach initially described by Shors et al. [17]. The idea of this approach is to compute the Hilbert transform of the cross-correlation function above. The maximum amplitude of this crosscorrelation corresponds to a 0-phase crossing point of its Hilbert transform. This 0 -phase point can be computed using linear interpolation from time samples in the vicinity and yields results with sub-millisecond accuracy.

\section{E. Delay confidence}

Both methods that use cross-correlation for delay computation have as a prerequisite that neighboring EGMs will be similar in morphology, and differ only by a time-shift. This is expected in the case in healthy myocardium, but may not be true in pathological situations.

To address this issue, we attach a confidence score $\mu_{i j}$ to each delay, estimated using the value of the cross correlation function.

$$
\mu_{i j}=\max _{\tau \in[0 ; T]} \frac{\int_{t=0}^{T}\left[f_{i}(t+\delta) f_{j}(t) d t\right]}{\int_{t=0}^{T} f_{i}(t) d t \int_{t=0}^{T} f_{j}(t) d t} \quad\left(\begin{array}{c}
f_{i}=s_{i} \text { or } \\
f_{i}=\frac{d s_{i}}{d t}
\end{array}\right)
$$

When this coefficient is high, the signals are similar in morphology, and delay computation is expected to be more robust.

\section{F. Merging information}

The previous signal processing steps allow us to compute IDT, estimated delays between neighboring points, and confidence indexes for both these markers. 
Merging of these different measures to create a spatially coherent activation map is equivalent to the solving the following system of linear equations:

$$
\left\{\begin{array}{c}
T_{i}=\widetilde{T}_{i} \\
T_{k}-T_{j}=\tilde{\tau}_{j k}
\end{array} \quad\left(i, j, k=1 \ldots N ; j<k ; k \in \Omega_{j}\right)\right.
$$

Where $\Omega_{j}$ is the $1^{\text {st }}$ order neighborhood of node $j$. In matrix form this system can be written as:

$$
\left[\begin{array}{l}
I \\
D
\end{array}\right] \mathrm{T}=\left[\begin{array}{l}
\widetilde{T} \\
\tilde{\tau}
\end{array}\right]
$$

Where $\mathbf{T}, \widetilde{\mathbf{T}}$ and $\tilde{\boldsymbol{\tau}}$ are the vector representations of activation times, IDT, and delay between neighboring nodes, and $\mathbf{D}$ is the incidence matrix of the underlying oriented graph corresponding to the mesh triangulation.

However, resolution of the previous equations using a leastsquares approach results in weighing all measures similarly. Since the nature of delay and IDT measures is different, a weighted least-squares approach was used. This enables us to give more weight to one or the other measurement/equation set. The resolution can then be formulated as:

$$
\mathbf{T}=\left(\left[\begin{array}{l}
\boldsymbol{I} \\
\mathbf{D}
\end{array}\right]^{t}\left[\begin{array}{cc}
(1-\lambda) \boldsymbol{I} & 0 \\
0 & \lambda \boldsymbol{I}
\end{array}\right]\left[\begin{array}{l}
\boldsymbol{I} \\
\mathbf{D}
\end{array}\right]\right)^{-1}\left[\begin{array}{l}
\boldsymbol{I} \\
\mathbf{D}
\end{array}\right]^{t}\left[\begin{array}{cc}
(1-\lambda) \boldsymbol{I} & 0 \\
0 & \lambda \boldsymbol{I}
\end{array}\right]\left[\begin{array}{l}
\widetilde{\mathbf{T}} \\
\widetilde{\tau}
\end{array}\right]
$$

Where $\lambda$ is a weighing scalar, ranging from 0 (IDT only) to 1 (delays only).

A more generic form of the previous formulation can also be used, with different weights $\mathrm{W}$ given to each individual measure/equation:

$$
\mathbf{T}=\left(\left[\begin{array}{l}
\boldsymbol{I} \\
\mathbf{D}
\end{array}\right]^{t} \mathbf{W}\left[\begin{array}{l}
\boldsymbol{I} \\
\mathbf{D}
\end{array}\right]\right)^{-1}\left[\begin{array}{l}
\boldsymbol{I} \\
\mathbf{D}
\end{array}\right]^{t} \mathbf{W}\left[\begin{array}{l}
\widetilde{\mathbf{T}} \\
\tilde{\boldsymbol{\tau}}
\end{array}\right]
$$

In an extension of the Gauss-Markov theorem, Aitken has shown that $\mathbf{T}$ becomes the best linear unbiased estimator when the weights $\mathrm{W}$ are related to the inverse of the variance of the measurement [18] given the underlying assumption that delays and IDT measures are heteroscedastic with respect to a measurable parameter. We therefore set:

$$
\mathbf{W}=\boldsymbol{I}\left[\begin{array}{c}
\operatorname{Var}\left(\tilde{T}_{i}\right)^{-1} \\
\operatorname{Var}\left(\tilde{\tau}_{j k}\right)^{-1}
\end{array}\right] \quad(i, j, k=1 \ldots N ; j<k)
$$

\section{G. Estimation of variance}

In this work, we hypothesized that activation time and delay estimates would be heteroscedastic with regards to the confidence scores previously defined. The variance of each measure was estimated using a standard 2 parameter log-linear model [19] from our confidence scores $\kappa_{i}$ and $\mu_{i}$ :

$$
\begin{gathered}
\log \left(\operatorname{Var}\left(\tilde{T}_{i}\right)\right)=C_{1} \kappa_{i}+C_{2} \\
\log \left(\operatorname{Var}\left(\tilde{\tau}_{i}\right)\right)=C_{3} \mu_{i}+C_{4}
\end{gathered}
$$

Model coefficients were estimated using non-linear leastsquares regression on the activation data from the in-silico dataset, for which the reference activation pattern was precisely known.

Testing for heteroscedasticity and its estimation by the above model was carried out using an $F$-test statistical framework, in a manner analogous to a Breusch-Pagan test [20].

\section{H. Activation map precision evaluation}

Root mean square errors between reference and computed activation times were computed. A paired $t$-test was used to compare the quality of each activation map using IDT and activation maps corrected using our workflow.

Local ( $1^{\text {st }}$ order neighbors) gradients in activation time were computed for each point. The root mean square of the error vector norm (RMSEN) was taken as a metric of AT gradient quality. A paired t-test was used to compare RMSEN as computed by the IDT method and our methodology.

All analyses were conducted using custom made software based on Matlab (The Mathworks, Inc., Natick, MA).

\section{MODEL RESULTS AND FIT}

\section{A. Low-pass filtering effect of ECGi}

Our results confirm the low-pass filtering effect of the methodology. Reconstructed signals had lower amplitude intrinsic deflections $(-0.39 \pm 0.20$ vs. $-1.10 \pm 0.89 \mathrm{~V} / \mathrm{s} ; \mathrm{p}<0.01)$ and longer intrinsic deflection durations $(17.7 \pm 12.1$ vs. $11.3 \pm 5.4 \mathrm{~ms} ; \mathrm{p}<0.01$ ) compared to reference data.

\section{B. AT maps and confidence score}

Standard activation maps using the intrinsic deflection of the signal for the estimation of ATs resulted in a mean error of $-0.2 \pm 14.7 \mathrm{~ms}$ for the 6 different activation sequences. Correlation was strong $(\mathrm{R}=0.93)$ and linear regression resulted in a slope of 0.98 showing good fit between the estimates and gold standard data (Figure 3A).

IDT estimates were heteroscedastic with regards to the confidence score described in part B of the Methods section (Figure $3 \mathrm{~B}-\mathrm{p}<0.001$ ).

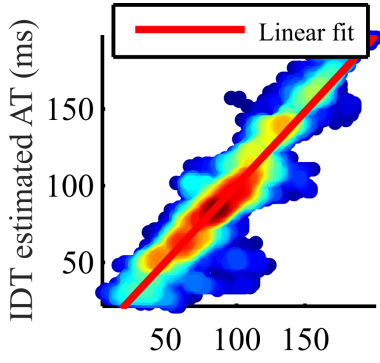

Reference AT (ms)

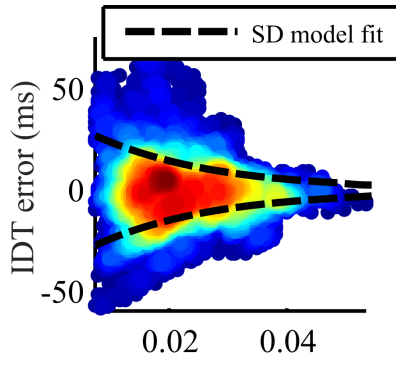

Confidence score
Figure 3: A) Comparison of IDT estimates with reference data B) Heteroscedastic error with respect to confidence score. Colors indicate point density. 
A

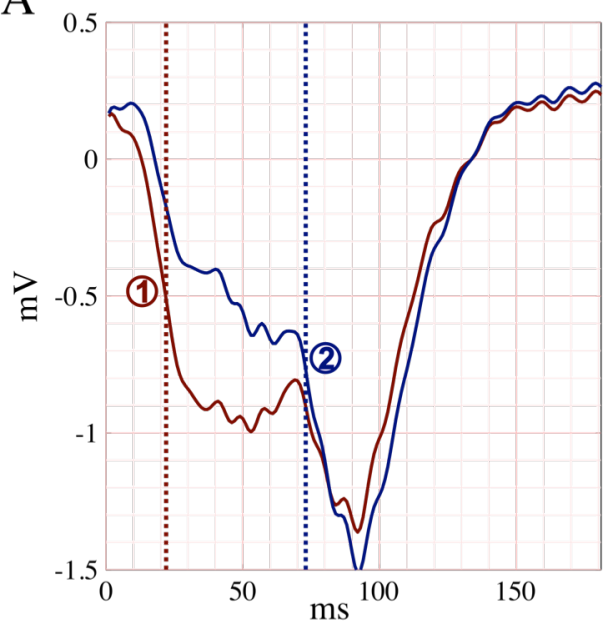

B

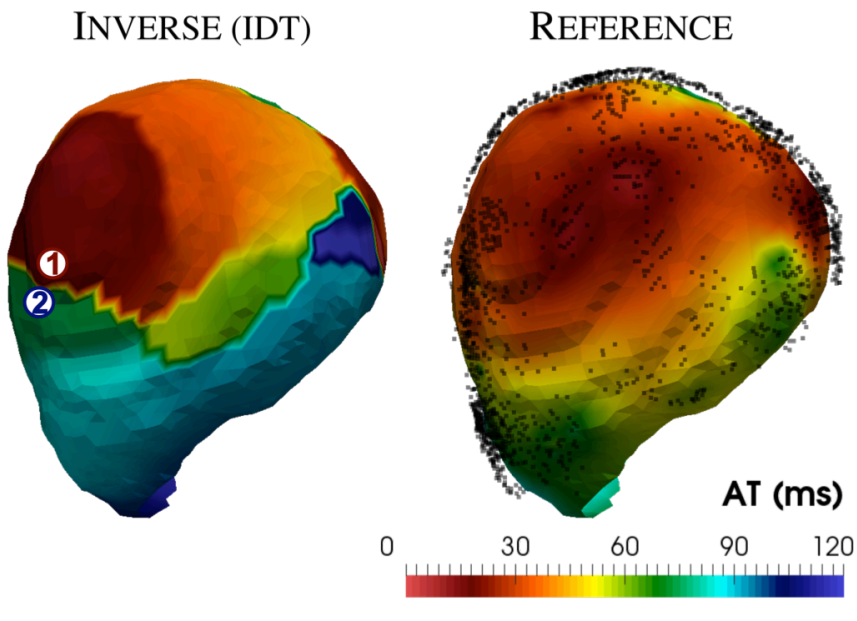

Figure 4: A) Reconstructed EGMs on both sides of an artificial line of block. IDT delay between the two points is 51ms (reference is 9ms). B) Example IDTinverse computed (left) and reference (right) AT maps. Numbered points correspond to EGMs in panel A. Measured point density is overlayed on the map on the right. Note how the overall EGM morphology is similar, but the marker abruptly jumps from one deflection to the next.

\section{Signal delay computation and confidence score}

Signal delays between neighboring points were computed using the 3 previously described approaches. Even though the general pattern of activation is reproduced as documented in the previous section, areas of strong IDT gradients are present, creating visually "patchy" activation maps (Figure 4).

These areas of strong gradients are absent in the reference dataset. They are artifacts of the overall inverse problem and activation map construction process.

The different methods of delay computation are compared in Table I.

TABLE I

COMPARISON OF DELAY COMPUTATION METHODS

\begin{tabular}{cccc}
\hline \hline & $\begin{array}{c}\text { IDT method } \\
\text { (Eq. 4) }\end{array}$ & $\begin{array}{c}\text { Signal correlation } \\
\text { maxima (Eq. 5) }\end{array}$ & $\begin{array}{c}\text { Signal derivative } \\
\text { correlation } \\
\text { maxima (Eq. 6) }\end{array}$ \\
\hline RMSE (ms) & 9.58 & 8.06 & 3.42 \\
$\begin{array}{c}\text { Correlation } \\
\text { coefficient (\%) }\end{array}$ & 29.2 & 32.1 & 66.7 \\
\hline \hline
\end{tabular}

Delay estimates and variance model fit using the signal derivative correlation maxima method is displayed in figure 5 . The slope of the linear fit of the delays against reference delays is 1.10 , leading to a small underestimation of the absolute delay values using this method.

Delay errors were heteroscedastic with respect to the correlation coefficient (Figure 5B $-\mathrm{p}<0.001$ ).

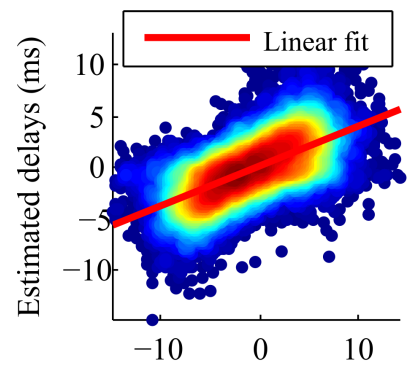

Reference delays time (ms)

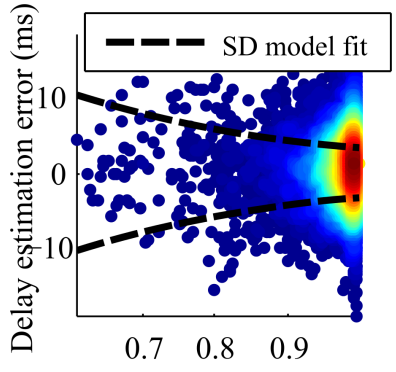

Confidence score $\mu$
Figure 5: A) Comparison of reference delays and those obtained using signal derivative correlation maxima. B) Confidence score, dispersion of error and model fit. Colors indicate point density.

\section{Merging information}

Resolution of the system using preferentially IDTs or delays (i.e. varying parameter $\lambda$ ) resulted in a U-shaped RMSE in terms of AT errors. The system cannot be solved if only delays are considered, since a reference time instant is lacking.

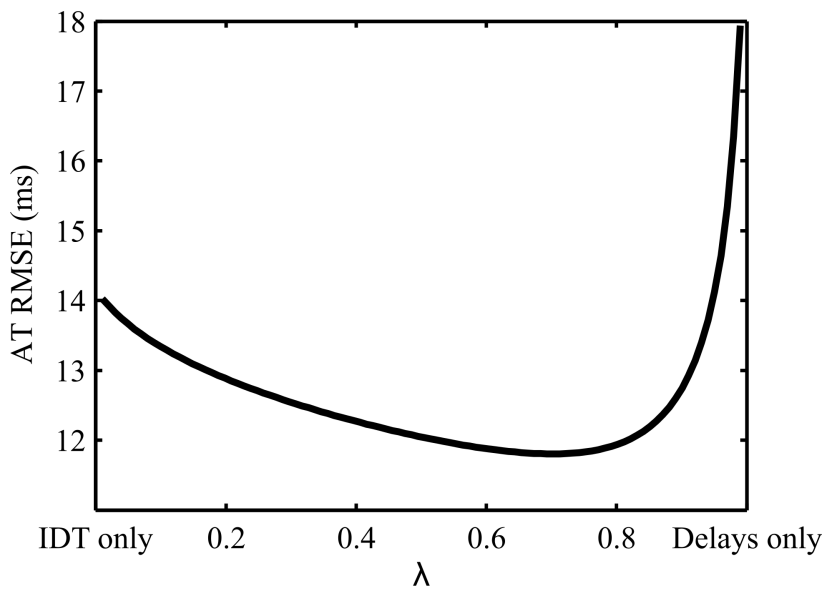

Figure 6: Reconstruction error as a function of $\lambda$ for a sample simulation. The U-shaped curve illustrates that combining both delays and IDTs is superior to either method alone. Note: System is underdetermined for $\lambda=1$.

The integration of delay measurements therefore improves the quality of the reconstruction from IDT only (corresponding to $\lambda=0$ ). A standard least squares resolution using equally weighted IDT and delays is equivalent to choosing $\lambda=0.5$ (Figure 6).

To further improve the reconstruction and make use of the confidence values described previously, we solved the weighted system using the strategy described in section III F.

This resulted in an RMSE in activation time over all simulations of $10.4 \pm 1.5$ vs. $14.4 \pm 3.6 \mathrm{~ms}$ for ITD alone ( $\mathrm{p}<$ $0.01)$. 


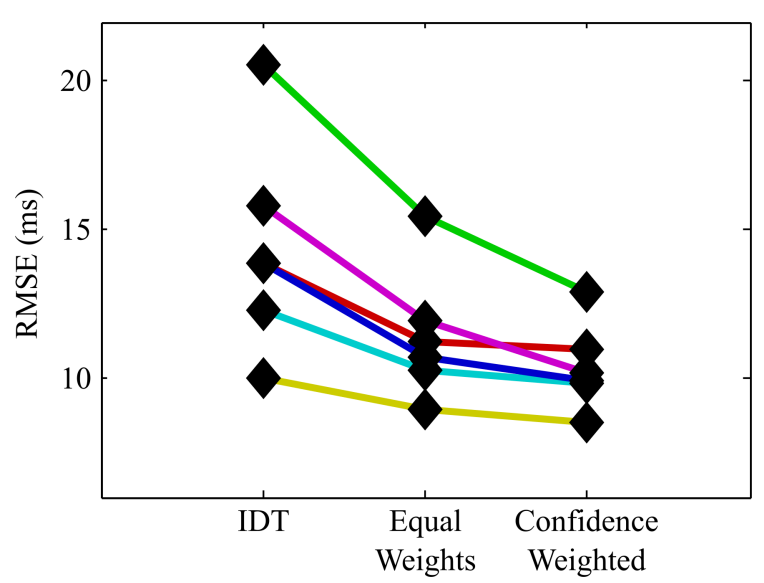

Figure 7: RMSE for the different simulations

RMSE for IDT estimates, standard least squares resolution method (equal weights), and the individual inverse varianceweighting scheme are presented in figure 7.

AT RMSE reduction using the new methodology is driven by the correction of artificial AT gradients.

\section{VALIDATION RESULTS}

\section{A. Activation map evaluation}

Results of the method on our validation clinical dataset were similar to those observed on the simulations. Example activation maps using the 3 methods on the same patient are presented in figure 8.

Spread in RMSE was more important than in the simulation cases. Mean RMSE using our method was $23.2 \pm 8.4$ vs. $28.6 \pm 10.1 \mathrm{~ms}$ using IDT only $(\mathrm{p}<0.01)$ representing a $19.3 \pm 10.6 \%$ relative error reduction. Importantly, enhancement of activation map precision was consistent over all patients (Figure 9).

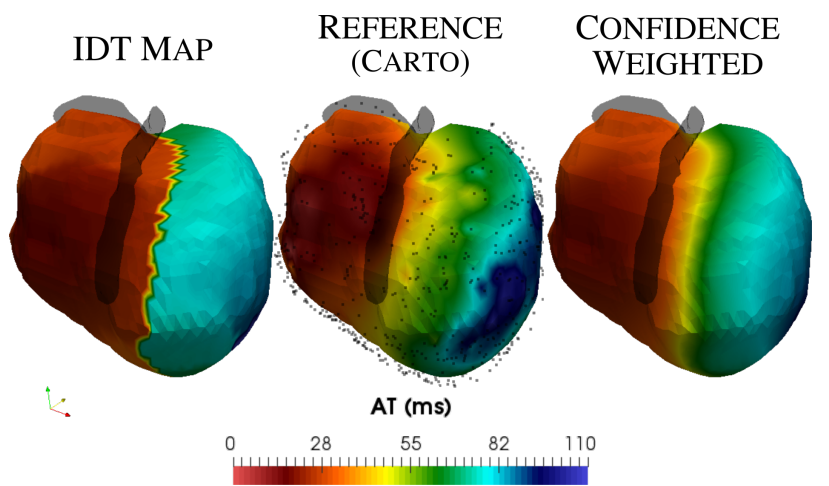

Figure 8: example enhancement of activation times using proposed method (anterior view - both ventricles). Long anterior descending artery is drawn in black (semi-opaque) for reference, along with point density on the reference map. An artificial "line of block" is introduced by the reconstruction process and IDT marker placement (center). The confidence-weighted map partially corrects the situation.

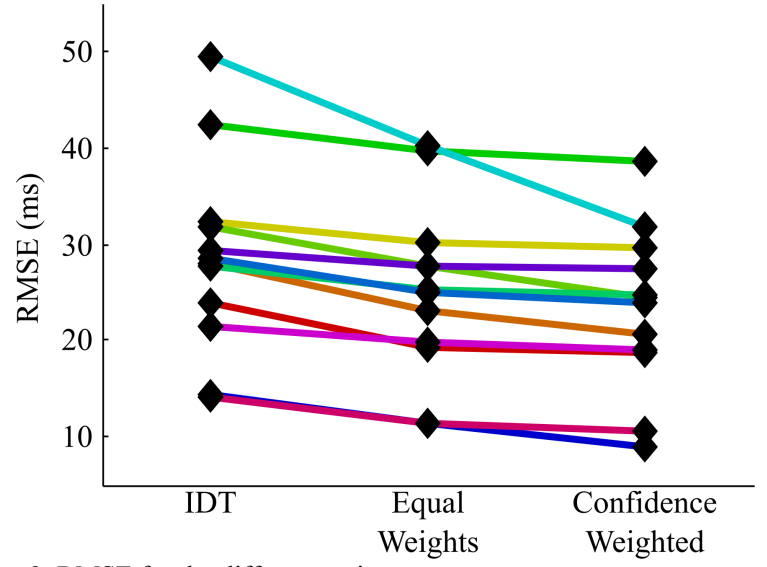

Figure 9: RMSE for the different patients

\section{B. Gradient maps evaluation}

Enhancement of gradient maps was also consistent with simulation data, with a mean RMSEN of $2.9 \pm 0.7 \mathrm{~ms}$ using our confidence-weighted method vs. $6.0 \pm 2.7 \mathrm{~ms}$ using the IDT only, representing a $47.2 \pm 13.7 \%$ relative error reduction. Enhancement was consistent for all patients (Figure 10).

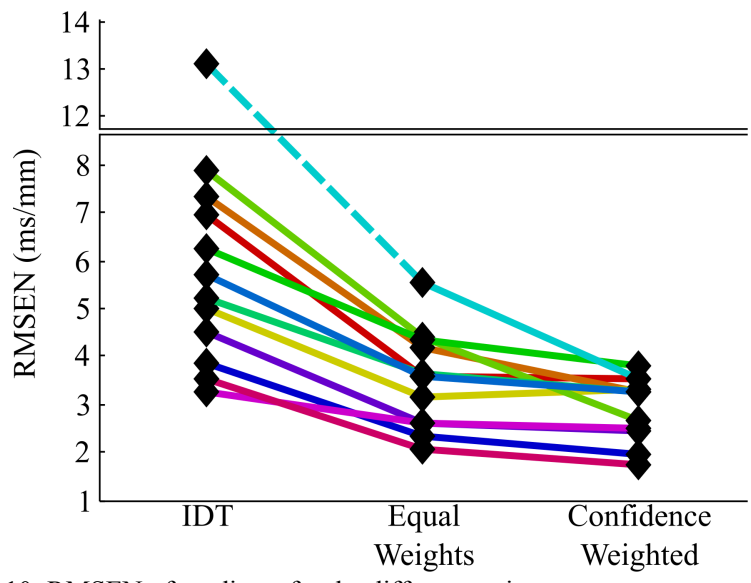

Figure 10: RMSEN of gradients for the different patients.

\section{DISCUSSION}

\section{A. Intrinsic deflection for activation time measurement}

This paper presents a methodology to construct activation maps from unipolar EGMs. The main objective is to build better maps in the particular context of ECGi, which computes a smoothed version of epicardial potentials. In this context, we have shown that using IDT as an AT marker is imprecise and leads to the appearance of false gradients. These limitations are nevertheless applicable to all unipolar signals in which myocardial activation is fragmented or the acquisition process leads to a spatial or temporal low pass filtering effect. This is the case in many situations: optical mapping studies [21] or invasive recordings with imperfect catheter contact for example.

Estimating delays between neighboring points is an interesting option to create more precise activation maps.

\section{B. Time delays between EGMS}

Estimating delays between neighboring points can also be useful per se and used to build a vector field representation of 
activation propagation velocity. Such an approach has been used by Morley et al. [22]. In their work, the authors use the intrinsic deflections to compute ATs and construct velocity vector fields.

Fitzgerald et al. [16] have constructed similar vector fields, albeit with signal cross-correlation. In our study, cross correlation of the signal's time derivative was the best estimate of delays between signals. Shors et al. [17] have demonstrated the potential of this approach on invasive measurements. We show that the combination of IDT and delays yields the best results.

The large dispersion of the IDT derived delays compared to reference (as illustrated in figure 5) indicates that on our model data, the inverse reconstruction and the marker positioning method combined often result in the appearance of artefactual activation time gradients. This situation is also illustrated by figure 8 . In this example, the reconstruction process creates an artificial line of conduction block on the anterior wall along the ventricular septum, just left of the long anterior descending artery. Although conduction slowing does exist in this area, it is strongly exaggerated by the reconstruction. Such lines of block have already been observed in other studies [23], [24] but should, in light of these results, be interpreted with caution.

\section{Validation}

Our results were applied to recordings obtained in a clinical setting. Although invasive epicardial mapping has already been used as a reference to evaluate ECGi [25], [26], this article presents the largest series ever published, which is an important step in in-vivo validation of the technique.

Compared to preclinical studies and our own modeling studies, clinical results show greater discrepancies between reference and reconstruction. We believe that three areas of concern arise in a clinical setting and may explain these results: (i) the importance and type of measurement/geometrical noise; (ii) the more complex activation sequences and electrophysiological substrate found in diseased human hearts; (iii) the imperfect nature of the reference dataset used for comparison.

The mean reconstruction error is more important in the clinical context than in simulations, and it is expected that the optimal coefficients $C_{1 \ldots 4}$ will be different in this setting. Nevertheless, the method significantly improves activation map precision compared to IDT alone, and also improves the maps constructed using equal coefficients for delays and ATs.

It is also important to note that the clinical data included ventricular paced or sinus rhythms, which are generally considered simple and are non-reentrant. Stable reentrant activity such as ventricular tachycardia can be mapped using a similar strategy in which the delay computation is estimated modulo the arrhythmia cycle length. The minimization problem becomes non-linear in this case but is still simple to solve. This consideration set aside, ventricular tachycardia creates simple activation patterns similar to those of paced ventricles, and complications are expected only at the VT isthmus, where the low voltage amplitudes make activation mapping complex.

The depicted method could also be used for atrial activation mapping, which suffers from similar artifacts. Compared to ventricular signals, atrial signals are lower amplitude. Recordings of this nature therefore have a less favorable signal to noise ratio regarding external interference. The other main differences between atrial and ventricular electrical activity are structural in nature. Atrial myocardium is thinner, has no specialized conduction system and exhibits greater anisotropy. These elements imply that adjacent myofiber bundles can be less tightly coupled, and atrial signals more variable, which will possibly make a delay-based method less relevant to optimize activation maps in the context of ECGi.

Areas of signal fragmentation are especially difficult to annotate in terms of activation times [27]. Standard markers on unipolar and bipolar signals often no longer correspond underlying tissue activation time, even in invasive recordings. These situations are often of clinical importance, corresponding to diseased tissue prone to reentry; and our method can provide an alternative annotation method that can be particularly useful.

The improvement in terms of activation time gradients is more important and could be expected given our methodology, which combines IDT measures with delay estimates. The important error in gradients observed using only IDT measures highlights our previous comment about interpretation of conduction block lines using this methodology.

\section{CONCLUSION}

ECGi is an effective tool for computing non-invasive activation maps from body surface potentials. Using the conventional methodology based on the timing of the IDT, activation time RMSE is $28.6 \pm 10.1 \mathrm{~ms}$ on our clinical dataset, and artificial lines of strong activation time gradient are created. Enhancement of activation maps can be obtained by estimating delays between neighboring points and combining this information with the IDT derived AT estimates.

The two measurement sets can be combined using a weighted least squares approach, in which measurement variances are estimated from the morphologies of the reconstructed signals.

The method significantly improved activation time estimates to a mean RMSE of $23.2 \pm 8.4 \mathrm{~ms}$.

\section{ACKNOWLEDGMENT}

The authors gratefully acknowledge Pr. Michel Haïssaguerre and his team of cardiac electrophysiology in Bordeaux, France for the patient datasets. This work was granted access to the HPC resources of IDRIS under the allocation x2015037379 made by GENCI.

\section{REFERENCES}

[1] D. P. Zipes and J. Jalife, Cardiac Electrophysiology: From Cell to Bedside. Saunders/Elsevier, 2009.

[2] Y. Wang and Y. Rudy, "Application of the method of fundamental solutions to potential-based inverse electrocardiography.," Ann. Biomed. Eng., vol. 34, no. 8, pp. 1272-88, Aug. 2006.

[3] C. N. Nowak, et al., "Spatial-temporal filter effect in a computer model study of ventricular fibrillation.," Biomed. Tech., vol. 53, no. 4, pp. 163-73, 2008.

[4] C. R. Johnson and R. S. MacLeod, “Adaptive local regularization 
methods for the inverse ECG problem.," Prog. Biophys. Mol. Biol., vol. 69, no. 2-3, pp. 405-23, Jan. 1998.

[5]

P. M. van Dam, et al., "Non-invasive imaging of cardiac activation and recovery.," Ann. Biomed. Eng., vol. 37, no. 9, pp. 1739-56, Sep. 2009.

B. He, et al., "Noninvasive three-dimensional activation time imaging of ventricular excitation by means of a heart-excitation model," Phys. Med. Biol., vol. 47, no. 22, pp. 4063-4078, Nov. 2002.

[7] L. Wang, et al., "Physiological-model-constrained noninvasive reconstruction of volumetric myocardial transmembrane potentials.," IEEE Trans. Biomed. Eng., vol. 57, no. 2, pp. 296-315, 2010.

[8] C. Han, et al., "Noninvasive three-dimensional cardiac activation imaging from body surface potential maps: a computational and experimental study on a rabbit model.," IEEE Trans. Med. Imaging, vol. 27 , no. 11, pp. 1622-30, Nov. 2008.

C. Han, et al., "Imaging cardiac activation sequence during ventricular tachycardia in a canine model of nonischemic heart failure," no. 10, pp. 108-114, 2015.

[10] Y. Rudy, "Noninvasive electrocardiographic imaging of arrhythmogenic substrates in humans.," Circ. Res., vol. 112, no. 5, pp. 863-74, Mar. 2013.

[11] K. H. W. J. ten Tusscher, et al., "A model for human ventricular tissue.," Am. J. Physiol. - Hear. Circ. Physiol., vol. 286, no. 4, pp. H1573- H1589, 2004.

[12] M. Potse, et al., "A comparison of monodomain and bidomain reaction-diffusion models for action potential propagation in the human heart.," IEEE Trans. Biomed. Eng., vol. 53, no. 12 Pt 1, pp. 2425-35, Dec. 2006.

[13] D. Krause, et al., "Hybrid Parallelization of a Large-Scale Heart Model," in Facing the Multicore-Challenge II: Aspects of New Paradigms and Technologies in Parallel Computing, LNCS Vol. 7174, Springer Berlin Heidelberg, 2012, pp. 120-132.

[14] D. Shepard, "A two-dimensional interpolation function for irregularly-spaced data," in Proceedings of the 1968 23rd ACM national conference, 1968, pp. 517-524.

[15] C. D. Cantwell, et al., "Techniques for automated local activation time annotation and conduction velocity estimation in cardiac mapping," Comput. Biol. Med., 2015.
[16] T. N. Fitzgerald, et al., "Estimation of cardiac conduction velocities using small data sets," Ann. Biomed. Eng., vol. 31, no. 3, pp. 250261, 2003.

[17] S. M. Shors, et al., "A method for determining high-resolution activation time delays in unipolar cardiac mapping," IEEE Trans. Biomed. Eng., vol. 43, no. 12, pp. 1192-1196, Dec. 1996.

[18] A. C. Aitken, "IV.-On Least Squares and Linear Combination of Observations.," Proc. R. Soc. Edinburgh, vol. 55, pp. 42-48, 1936.

[19] R. J. Carroll and D. Ruppert, Transformation and Weighting in Regression. Taylor \& Francis, 1988.

[20] T. S. Breusch and A. R. Pagan, "A Simple Test for Heteroscedasticity and Random Coefficient Variation," Econometrica, vol. 47, no. 5, pp. 1287-1294, Sep. 1979.

[21] R. D. Walton, et al., "A novel approach for deriving global activation maps from non-averaged cardiac optical signals.," 35th Annu. Int. Conf. IEEE Eng. Med. Biol. Soc., vol. 2013, pp. 1772-5, 2013.

[22] G. E. Morley, et al., "Characterization of conduction in the ventricles of normal and heterozygous $\mathrm{Cx} 43$ knockout mice using optical mapping.," J. Cardiovasc. Electrophysiol., vol. 10, no. 10, pp. 1361-75, Oct. 1999.

[23] A. Auricchio, et al., "Characterization of Left Ventricular Activation in Patients with Heart Failure and Left Bundle-Branch Block," Circulation, vol. 109, no. 9, pp. 1133-1139, 2004.

[24] N. Varma, "Left ventricular electrical activation during right ventricular pacing in heart failure patients with LBBB: Visualization by electrocardiographic imaging and implications for cardiac resynchronization therapy," J. Electrocardiol., vol. 48, no. 1, pp. 53-61, 2015.

[25] J. L. Sapp, et al., "Inverse solution mapping of epicardial potentials: Quantitative comparison with epicardial contact mapping," Circ. Arrhythmia Electrophysiol., vol. 5, no. 5, pp. 1001-1009, 2012.

[26] R. N. Ghanem, et al., "Noninvasive Electrocardiographic Imaging (ECGI): Comparison to intraoperative mapping in patients," Hear. Rhythm, vol. 2, no. 4, pp. 339-354, Apr. 2005.

[27] F. Del Carpio Munoz, et al., "Teaching Points With 3-Dimensional Mapping of Cardiac Arrhythmias: Taking Points: Activation Mapping," Circ. Arrhythmia Electrophysiol., vol. 4, no. 3, pp. e22e25, Jun. 2011. 